Review Article

\title{
Nano drug delivery systems: Targeted and site specific tool for therapeutic management
}

\author{
Ashish Garg', Vaibhav Tiwari ${ }^{2}$ \\ ${ }^{1}$ Department of P.G. Studies and Research in Chemistry and Pharmacy, Rani Durgavati Vishwavidyalaya, Jabalpur, 482001, \\ M.P., India
}

${ }^{2}$ Shri Shankarachrya Institute of Pharmaceutical Sciences and Research, Junwani, Bhilai, C.G., India

Received: 4 April 2019

Revised: 22 May 2019

Accepted: 30 May 2019

\begin{abstract}
Objective: Systemic drug delivery strategies like oral or canal administration of free medication possess comparatively low treatment potency and marked adverse aspect effects. The utilization of nanoparticles for drug delivery in most cases well enhances drug effectiveness, improves pharmacology and drugs unleash and limit their aspect effects. However, more improvement in drug effectiveness and vital limitation of adverse aspect effects may be achieved by specific targeting of nanocarrier-based delivery systems particularly together with native administration. Conclusion: In this review describes major blessings and limitations of organic and inorganic nanocarriers or living cell-based drug and super-molecule delivery systems. Among these, totally different nanoparticles, supramolecular gels, therapeutic cells as living drug carriers etc. have emerged as a replacement frontier in fashionable drugs.
\end{abstract}

Keywords: Drug targeting, nanoparticles, supramolecular gel, cell-based carriers, cancer

\section{Introduction}

It is well known that the main goal of drug delivery is effective and safe delivery of a drug to its target tissues, organs and cells. However, transport of a drug to its specific targets represents one of the major challenges for an effective drug delivery (Mainardesand Silva, 2004). Conventional enteral or parenteral drug delivery methods of systemic drug administration include the formulation of a drug into suitable dosage forms for oral/gastrointestinal administration (e. g. tablets, capsules, solutions, suspensions, syrups, etc.) or sterile drug preparations (solutions, suspensions, emulsions, or reconstituted lyophilized powders) suitable for administration by injection (intravenous, epidural, intramuscular, subcutaneous, etc.) (Robinson and Mauger, 1991). Nevertheless, these systemic drug delivery methods are associated with several limitations such as relatively low site

\footnotetext{
*Address for Corresponding Author:

Ashish Garg

Department of P.G. Studies and Research in Chemistry and Pharmacy, Rani Durgavati Vishwavidyalaya, Jabalpur, 482001, M.P., India

E-mail: ashish.garg071010@gmail.com
}

specific bioavailability of administered drugs, unfavorable distribution of drugs throughout the body and, in most cases, low accumulation in the target site, adverse side effects, etc. (Bardal et al., 2011). Besides, these drug delivery systems can't fulfill the ever-growing need of the modern medicine such as personalized medicine and targeted therapies. Therefore, there was an urgent need to develop novel drug delivery systems for localized and targeted delivery of therapeutics. Over the decades, researchers have extensively studied development of carrier-based drug delivery systems with truly targeted features towards specific disease as well as to prevent drug degradation, resistance, drug side effects and also improve the potential of individualized medicine. Various surface markers and cytokines such as integrins, folate, growth factors etc., which are known to express on disease cells and their microenvironments, were explored for developing counter marker functionalized drug carrier to recognize the target disease cells. Carrier-based targeted and localized drug delivery systems, e.g. supramolecular gels (Oneto et al., 2016), nanocarriers such as different types of nanoparticles (Garbuzenko et al., 2014; Chen et al., 2010), liposomes (Zhang et al., 2012; Saad et al., 2008),

DOI: https://doi.org/10.31024/apj.2019.4.3.2

2456-1436/Copyright (C) 2019, N.S. Memorial Scientific Research and Education Society. This is an open access article under the CC BYNC-ND license (http://creativecommons.org/licenses/by-nc-nd/4.0/). 
nanostructures lipid carriers (Taratula et al., 2013), dendrimers(Taratula et al., 2009; Taratula et al., 2011), quantum dots (Savla et al., 2011), live cells as living drug carriers (Fliervoet and Mastrobattista, 2016), micelles (Wan et al., 2019), polymeric nanoparticles (Duong et al., 2017), emulsions and microemulsions (Sahle et al., 2013), etc. were widely explored in the past few years. Such targeted drug delivery systems not only lead to accumulation of drug in the specific organs, tissues or cells resulting in increased treatment efficacy, but also reduce the drug availability into other organs thereby decreasing adverse side effects. To keep a distinctive perspective, contributions describing drug delivery through polymeric system, micelles, emulsions have been already described in corresponding reviews and are out of the scope of this review. This review will present the advances of various carrier based targeted drug delivery systems and provide their perspectives on future directions.

\section{Targeted drug delivery}

Targeting a drug delivery system to the specific organs, cells or cell organelles increases the accumulation of the delivered active components in the desired site of action and limits their accumulation in the healthy organs, tissues and cells. This in turn enhances the efficacy of the treatment and prevents severe adverse side effects upon the healthy organs and tissues. Previously, we proposed to subdivide all possible types of drug targeting on two major categories: passive and active targeting (Figure 1) (Minko et al., 2004). Socalled passive targeting relies on specific conditions in an entire organ or tissue which led to the accumulation of a drug delivery system with a specific size, molecular mass, charge, etc. In the case of cancer, it was found that high molecular weight drugs are preferentially accumulated in the solid tumors. Such situation was termed by Maeda and co-workers as enhanced permeability and retention (EPR) effect and results from the increased permeability of tumor vasculature in combination with limited lymphatic drainage (Maeda et al., 2017). However, such passive targeting is effective only when the diseased cells are localized in a relatively compact structure (e.g. solid tumor in case of cancer) and will not work if the affected cells are spread over the entire body (e.g. cancer metastases). In addition, passive targeting approach may utilize specific conditions in the diseased tissues or cells (e.g. low $\mathrm{pH}$ ) or the local delivery of active components specifically to the affected organ or tissue (e.g. topical delivery to skin or inhalation for lung delivery).

\section{Targeting ligands for cancer cells}

Probably, the one of the first and most widely used targets for the delivery of drugs, nucleic acids and other therapeutics specifically to cancer cells are folate (Dhawan et al., 2013). Folate receptors are overexpressed in most types of cancer cells. Consequently, conjugation of a ligand to a folate receptor with an anticancer drug or, most efficiently, to a drug delivery system, will provide for an active targeting of a payload explicitly to cancer cells. Folic acid and anti-folate receptor antibodies are the most widely used ligands to folate binding proteins on the surface of cancer cells. Antibodies to specific cancer cells or their fragments are also being widely used directly as therapeutic agents against various cancers or as targeting moieties for drug delivery systems (Alibakhshi et al., 2017). However, the lack of specificity of folate-based ligands and possible adverse side effects of antibodies as xenobiotics limit their

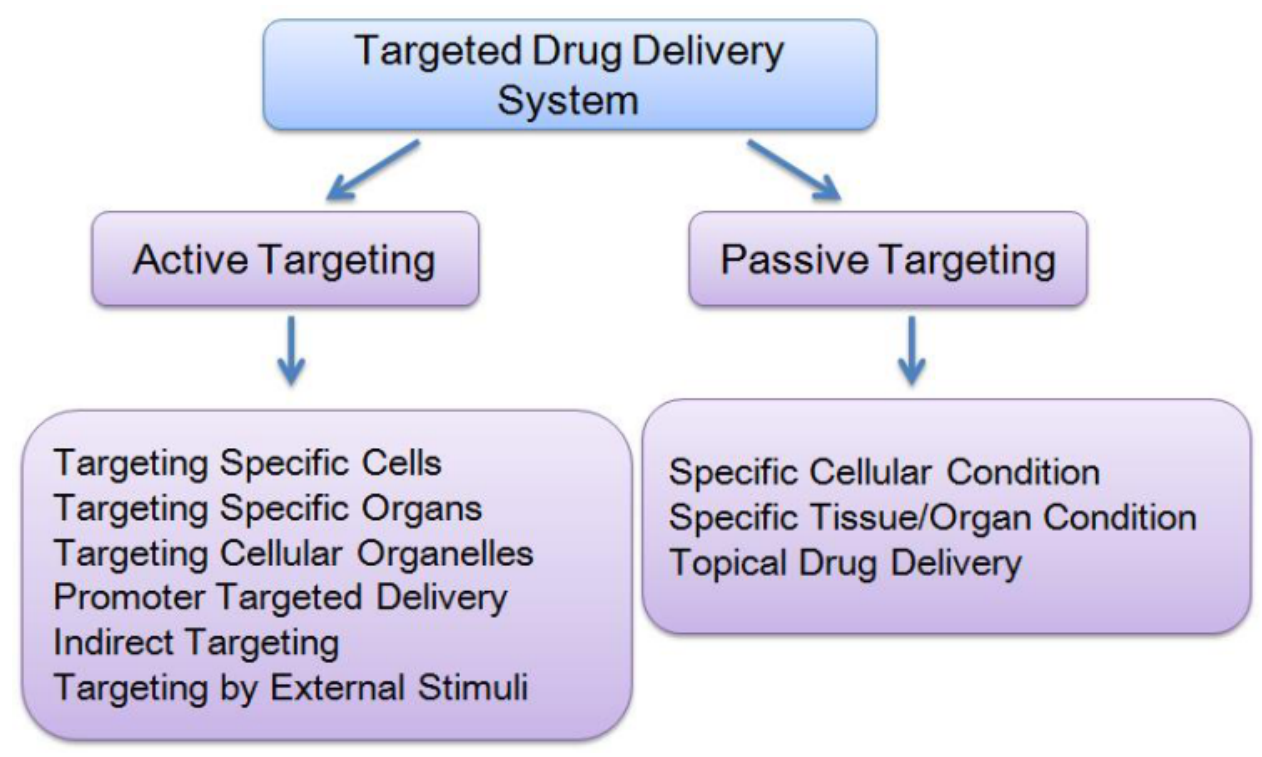

Figure 1. Schematic representation of targeted drug delivery system 
clinical use for cancer targeting of drugs or nucleic acids (Cruz et al., 2019). Peptide receptors represent another promising object for active targeting of drug delivery to cancer cells. Many peptide ligands (somatostatin analogs, vasoactive intestinal peptide, gastrin-releasing peptide, cholecystokinin/gastrin, neurotensin, substance $\mathrm{P}$, neuropeptide $\mathrm{Y}$, luteinizing hormone-releasing hormone - LHRH, bombesin, etc.) for these receptors have been used as targeting moieties for a specific delivery of drugs and nucleic acids to cancer cells (Engel et al., 2016). The work pioneered in the use of LHRH peptide for targeting of its receptors in different cancer cells and found that receptors specific to LHRH peptide overexpressed in many types of cancer cells and do not express in cells from visceral organs (Minko et al., 2013). One interesting finding was revealed in laboratory related to the use of LHRH peptide for targeting different nanoparticles to cancer. As expected, the conclusion found differences in organ distribution of non-targeted nanoparticles. However, the addition of LHRH peptide as a targeting moiety to all studied and protein-based nanoparticles, etc.; inorganic- gold, mesoporous silica nanoparticles leveled off these differences. All different nanocarriers (polymers, dendrimers, liposomes) targeted with LHRH peptide were equally efficient for tumorspecific treatment and imaging.

\section{Nanoscalebased localized and targeted delivery systems}

Nanoscale drug delivery is an integral part of the drug delivery systems. This type of system encapsulates drug or other therapeutically active components and carries them to its target site. Recently, nanoscale-based drug delivery has attracted considerable attention due to their wide applications in the targeted delivery, controlled release of drugs, as carriers for DNA, RNA in gene therapy etc. (Panyam et al., 2003). According to the shape of nanoparticles, they include dendrimer nanoparticles, den-dron nanostructures, nanovesicles, micellar nanoparticles, spherical nanoparticles, nanocrystals, nanobombs, nanoshells, nanocages, nanotubes, and nanorods. According to the chemical property of nanoparticles, three main nanoparticle families can be dis-tinguished: inorganic nanoparticles such as carbon nanotubes, iron oxide magnetic nanoparticles, silica nanoparticles, gold nanoparticles (GNPs), colloidal semiconduc-tor quantum dots, organic nanoparticles such as polymer nanoparticles, dendrimer nanoparticles, polymeric micelles, liposomes, and hybrid nanoparticles made from both organic and inorganic nanocomponents. To date, a large number of nanoscale drug delivery systems (organic and inorganic) have been developed and investigated. Organic and inorganic nanoparticles represent materials of multiple dimensions that demonstrate unique physical and chemical properties dependent on size, shape and composition. Organic and inorganic nanocarriers include but are not limited to: organic-dendrimers, polymeric micelles, liposomes, solid lipid and proteinbased nanoparticles, etc.; inorganic- gold, mesoporous silica, superparamagnetic iron oxide and paramagnetic nanoparticles, quantum dots, etc (Figure 2).

This includes the first Food and Drug Administration (FDA) approved liposomal nanoparticles "Doxil", which was known for encapsulating doxorubicin and its use in treatment of various cancers. In past few years, researchers paid significant attention to develop various types of nanocarrier-based systems for targeted drug delivery application. Here, we will summarize the advances and

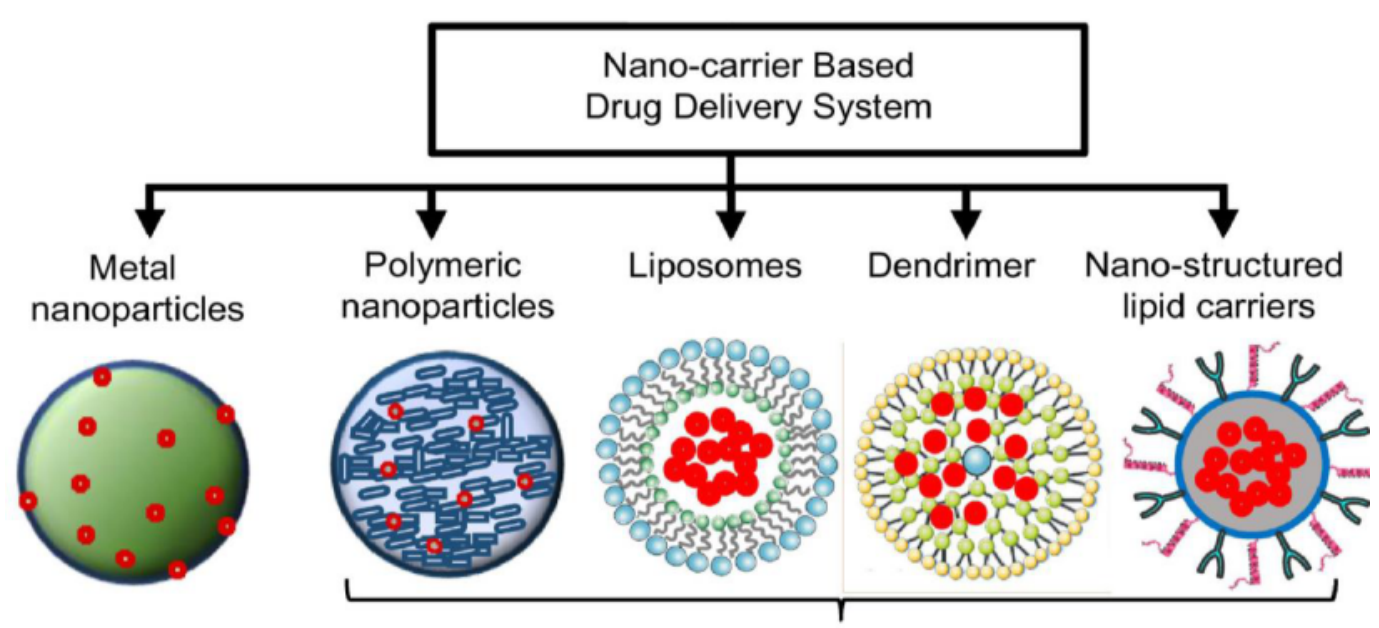

Inorganic nanoparticle

Organic (polymeric and lipid) nanoparticle

Figure 2. Classification of nanocarrier based drug delivery 
limitations of such nanocarrier-based organic and inorganic systems which are known to encapsulate, protect, and target therapeutics towards the disease site. Many advantages are associated with nanoparticles for tumor drug delivery. (i) they can protect the active substances from biodegradation by the surrounding medium and release the active substances when needed. For example, drugs can be prevented from being cleaved by enzymes [e.g., small interfering RNA (siRNA) by RNases in the plasma, proteins by pepsin or trypsin in the stomach] when encapsulated in nanoparticles. (ii) nanoparticles can increase the bioavailability of some antitumor compounds by increasing their water solubility, in vivo stability, penetrating capability of biological barriers and thus improve the pharmacokinetic profile. (iii) nanoparticles can also be designed to release their payload upon a trigger, resulting in stimuli- sensitive nanotherapeutics. In this way, increased specific uptake reduces the probability for unspecific uptake and MDR/ATP efflux pump-driven elimination and thus increases the antitumor efficacy of drugs. (iv) many nanoparticles have the characteristics of good storage stability, biocompatibility, and simple convenient preparation. The phenomenon of drug targeting towards cancer site via nanocarriers express in figure 3 .

Liposomes: Liposomes are a form of vesicles that consist either of many, few or just one phospholipid bilayers. The polar character of the liposomal core enables polar drug molecules to be encapsulated. Amphiphilic and lipophilic molecules are solubilised within the phospholipid bilayer according to their affinity towards the phospholipids. Participation of nonionic surfactants instead of phospholipids in the bilayer formation results in niosomes. Channel proteins can be incorporated without loss of their activity within the hydrophobic domain of vesicle membranes, acting as a size-selective filter, only allowing passive diffusion of small solutes such as ions, nutrients and antibiotics. Thus, drugs that are encapsulated in a nanocage-functionalized with channel proteins are
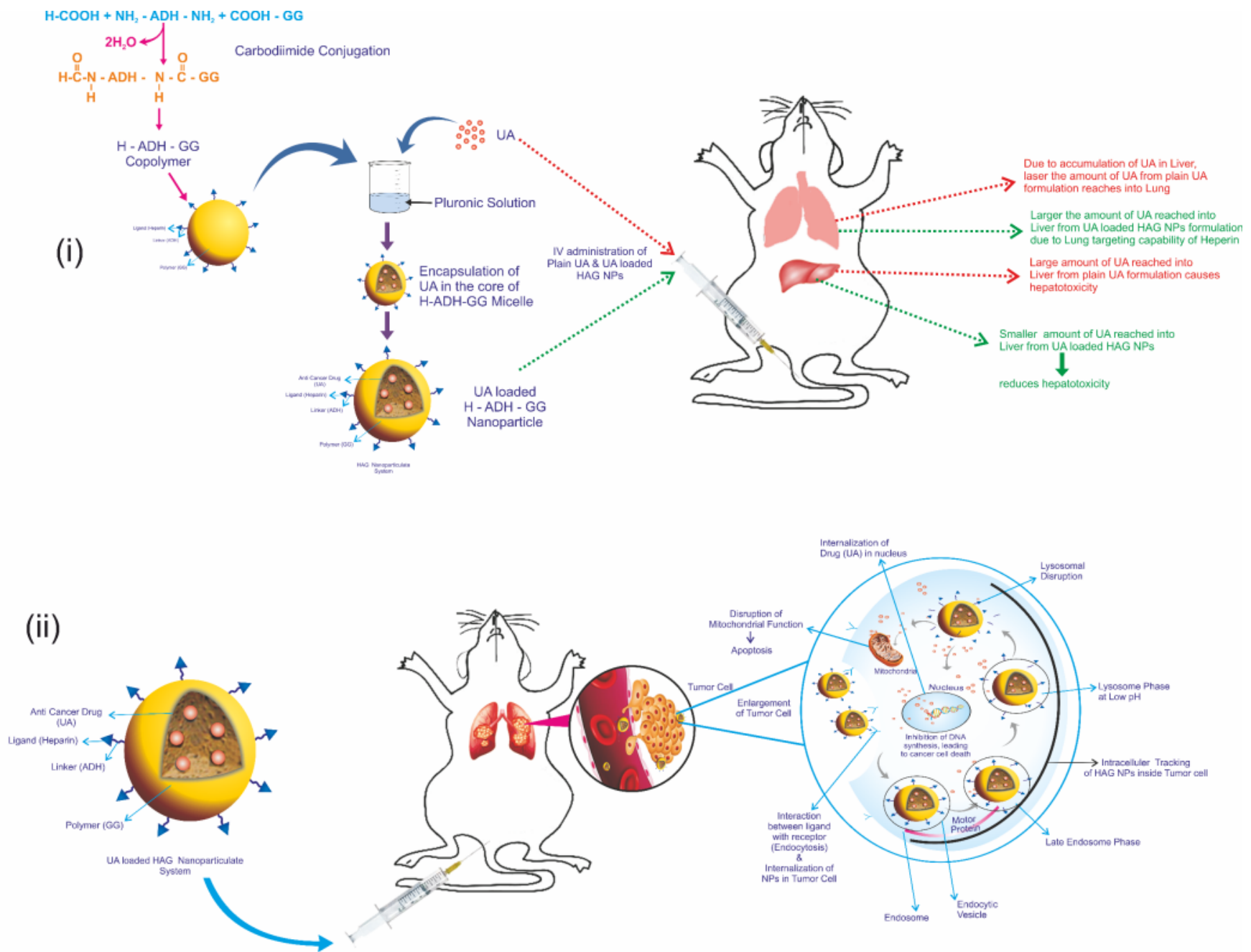

Figure 3. Targeting of anticancer drug containing nanoparticulate system against tumor cell (Garg et al., 2019). 


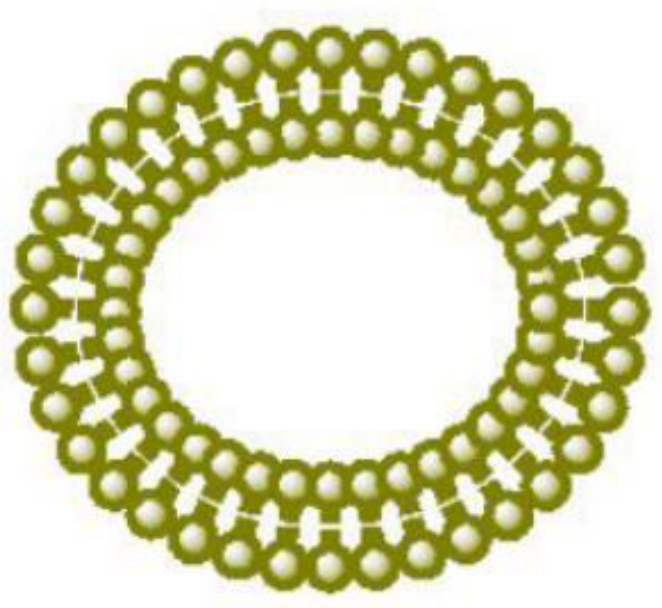

Figure 4. Structure of Liposome (Garg et al., 2017)

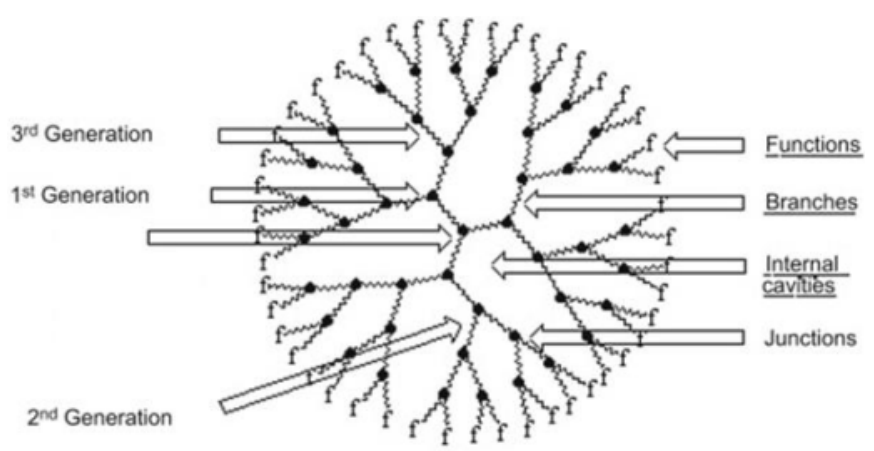

$4^{\text {th }}$ Generation Dendrimer

Figure 5.Structure of Dendrimer with their functionality (Wayne, 2013).

effectively protected from premature degradation by proteolytic enzymes. The drug molecule, however, is able to diffuse through the channel, driven by the concentration difference between the interior and the exterior of the nanocage (Sharma, 1997) (Figure 4). Liposome-based anticancer therapeutics such as DaunoXome, Myocet, VincaXome, DepoCyt, and Caelyx are currently available in the market for clinical use and many new liposomal formulations have been explored for clinical trials (Bulbake et al., 2017).

Dendrimers: Dendrimers (Figure 5) are nanometer-sized, highly branched and monodisperse macromolecules with symmetrical architecture. They consist of a central core, branching units and terminal functional groups. The core together with the internal units, determine the environment of the nanocavities and consequently their solubilizing properties, whereas the external groups the solubility and chemical behaviour of these polymers. Targeting effectiveness is affected by attaching targeting ligands at the external surface of dendrimers, while their stability and protection from the Mononuclear Phagocyte System (MPS) is being achieved by functionalization of the dendrimers with polyethylene glycol chains (PEG). Liquid Crystals combine the properties of both liquid and solid states. They can be made to form different geometries, with alternative polar and non-polar layers (i.e., a lamellar phase) where aqueous drug solutions can be included.

Nanoparticles: Nanoparticles (including nanospheres and nanocapsules of size $10-200 \mathrm{~nm}$ ) are in the solid state and are either amorphous or crystalline. They are able to adsorb and/or encapsulate a drug, thus protecting it against chemical and enzymatic degradation. In recent years, biodegradable polymeric nanoparticles have attracted considerable attention as potential drug delivery devices in view of their applications in the controlled release of drugs, in targeting particular organ/or tissue, as carriers of DNA in gene therapy, and in their abilities to deliver proteins peptides and genes through peroral route (Aminabhavi, 2001).Three kinds of polymers, polyesters, poly-alkyl-cyanoacrylates, and amphiphilic block copolymers, are usually used for the preparation of nanoparticles. Polyester belongs to biodegradable polymers, mainly including PLA, PLGA, and PCL. With the same molecular weight, PCL generally has the longest biodegradation half-life, whereas PLGA has the shortest biodegradation half-life. However, the ratio of lactic acid to hydroxylacetic acid in PLGA can be tuned to adjust its degradation performance. Amphiphilic block copolymers refer to copolymers having both a hydrophilic group and a lipophilic group in the polymer molecule. The diblock copolymers are the most common. Triblock copolymers and tetrablock copolymers also exist. PEGylated polyesters, including polyethylene glycol (PEG)-PLA, PEG-PLGA, PEG-PCL, and PEGylated poly-alkyl-cyanoacrylate, including PEG-PBCA and PEG-PiBCA, are common diblock copolymers. These copolymers have hydrophilic PEG chains, which can increase the stability of nanoparticles and prolong their circulation in blood.

Inorganic nanoparticles: Inorganic nanoparticles have good application prospects in the field of reaction catalysis, medicine, electromagnetic function, material modification, and so on (Gil et al., 2010). Several typical inorganic nanoparticles, including Gold NPs (GNPs) and iron oxide nanoparticles, are dis-cussed in the following sections. GNPs refer to gold particles with diameter from 5 to $300 \mathrm{~nm}$ and with the shape of sphere, shell, cage, and rod (Zheng et al., 2016). The GNP suspensions are usually presented in 
different colors from red to blue/purple because of the tunable size and the sur-face plasmon resonance phenomena caused by absorption of light. For small $(30 \mathrm{~nm})$ monodisperse GNPs, the absorption of light is located in the blue-green region of the spectrum $(450 \mathrm{~nm})$ while red light $(700 \mathrm{~nm})$ is reflected, resulting in solutions with a rich red color. As particle size increases, the wavelength absorption shifts to longer, redder wavelengths, and blue light is reflected, resulting in solutions with a pale blue or purple color. Uncoated GNPs are susceptible to aggregation in solution and can melt under laser irradiation. The GNPs are unstable in salt solutions. When excess salt is added to the GNP solution, the surface charge of GNPs becomes neutral, causing nanoparticle aggregation. As a result, the solution color changes from red to blue. GNPs exhibit unique physicochemical properties including their ability to bind amine and thiol groups, allowing easy surface functionalization and bioconjugation (Sharma et al., 2009). For example, thiolated PEG can bind with GNPs via $\mathrm{Au}-\mathrm{S}$ bonds, which forms thiolate protecting GNPs, increasing the stability of GNPs, and producing a hydrated PEG barrier to sterically hinder the attachment of phagocytes and reduce reticuloen-dothelial system uptake. Tumor-specific ligands can be grafted onto GNPs to increase the specificity and likelihood of drug delivery.

Iron oxide nanoparticles are iron oxide particles with diameters between 1 and $100 \mathrm{~nm}$. Three most common forms of iron oxides in nature are magnetite (Fe3O4) and its oxidized forms maghemite $(\gamma$-Fe2O3) and hematite $(\alpha$-Fe2O3) (Laurent et al., 2008). Magnetite has an inverse spinel structure, with oxygen forming a face-centered cubic crystal sys-tem. There are various shapes of iron oxides, such as nanorods, porous spheres, nanohusks, nanocubes, distorted cubes, and self-oriented flowers. Iron oxide nanoparticles with the size $<10-20 \mathrm{~nm}$ exhibit an inimitable form of magnetism, which is called superparamagnetism (Kodama et.al., 1999). Iron oxide magnetic nanoparticles are prepared by various methods, such as wet chemical techniques, dry processes, or microbiological techniques. Iron oxide nanoparticles with bare surface tend to aggregate because of strong magnetic attraction among particles, van der Waals forces, and high surface energy. A high concentration of local $\mathrm{Fe}$ ions is also toxic to organisms from $\mathrm{Fe}$ dissolution. Nontoxic, stable, and biocompatible magnetic nanoparticles can be achieved by coating them with organic or inorganic molecules, including silica, gold, phospholipids, fatty acids, polysaccharides, peptides, polymers. Iron oxide nanoparticles have gained increasing attention because of their unique properties, such as superparamagnetism, high surface-tovolume ratio, and easy separation capability (Xie et al., 2010). By locally applying an external magnetic field, iron oxide nanoparticles can be guided to specific tissues leading to nanoparticles accumulation, which can be used for targeting drug delivery to tumors. Moreover, iron oxide nanoparticles loaded with antitumor drugs have additional advantages over conventional antitumor drugs because of their visualization in MRI, high efficiency in drug targeting, and fewer side effects in normal tissues (Orel et al., 2015). Iron oxide nanoparticles can also be used in magnetic hyperthermia therapy or photothermal therapy as tumor therapeutics. In this method, iron oxide nanoparticle dispersion is directly injected into the tumors or administered into the vein followed by external magnetic field guidance. Then, the target tumor tissues are heated up by an alternating high-frequency magnetic field or an input of NIR radiation. The high temperature produced by the nanoparticles destroys surrounding tumorous cells inside tumors (Ling et al., 2015).

Carbon nanotubes: Carbon nanotubes (Figure 6) can be modified to circulate well within the body. Such modifications can be accomplished with covalent or non covalent bonding. Modifications can increase or decrease circulation time within the body. Carbon nanotubes no significant toxicity when they have modified so as to be soluble in aqueous body type fluids. They enter readily into the cells. Cancer cells in tumor are larger than normal cells and also exhibit leakage. Large molecules which circulate slowly can leak into and accumulate in cancer cell. Carbon nanotubes carrying active agents have been demonstrated in animal studies to do this. Researches have also used carbon tubes to deliver the precursors of active drug which they call a prodrug. eg: Cisplatin (Manivannan, 2010).

Transdermal Drug Delivery System: Transdermal drug delivery is defined as self contained, discrete dosage forms which, when applied to the intact skin, deliver the drug, through the skin at controlled rate to the systemic

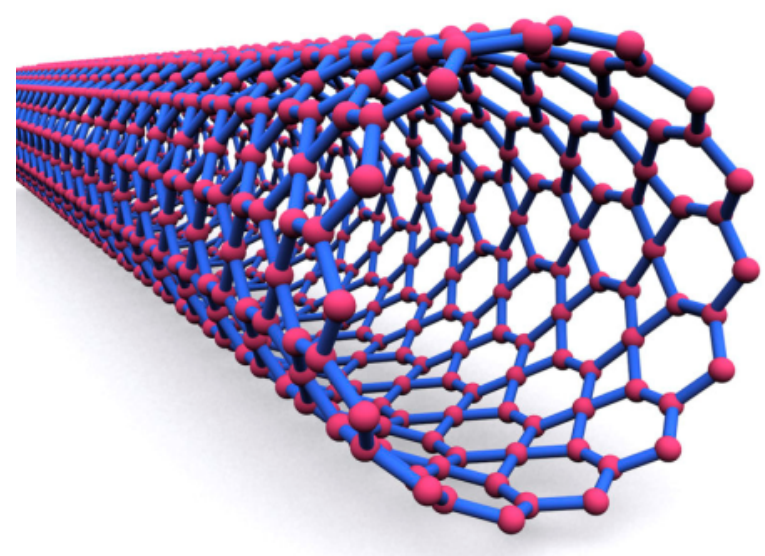

Figure 6. Carbon nanotube (single walled) (Han et al., 2017). 
circulation. Transdermal drug delivery system (TDDS) established itself as an integral part of novel drug delivery systems (Arunachalamet al., 2010). Delivery via the transdermal route is an interesting option because transdermal route is convenient and safe.

The positive features of delivery drugs across the skin to achieve systemic effects are:

$\checkmark$ Avoidance of first pass metabolism

( Avoidance of gastro intestinal incompatibility

4 Predictable and extended duration of activity

Improving physiological and pharmacological response

(1) Termination of therapy is easy at any point of time

G Greater patient compliance due to elimination of multiple dosing profile

(1) Provide suitability for self administration

Enhance therapeutic efficacy

Microemulsion: Microemulsions have been studied for delivery of Anti hypertensive drug such as lacidipine (LCDP), a poorly water soluble and low bioavailable drug(Zhang et al., 2012). The bioavailability studies in rabbits showed that about 3.5 times statistically significant $(\mathrm{p}<0.05)$ improvement in bioavailability, after transdermal administration of microemulsion gel compared to oral suspension. Microemulsion based transdermal therapeutic system of LCDP was developed and optimized using Box-Behnken statistical design and could provide an effective treatment in the management of hypertension. IPM (isopropyl myristate) containing 10\% (v/v) DMF (dimethyl Formamide) was selected as oil phase to solubilize LCDP that could further maintain large concentration gradient towards skin. The solubility of LCDP found to be 42.83 in IPM containing 10\% (v/v) DMF (Mendes et al., 2019). LCDP is released and permeated well from microemulsion gel by transdermal route, as compared to the oral suspension. This difference was because of stratum corneum that could delay the permeation of LCDP from microemulsion gels in contrast, suspension administered by oral route is an immediate release dosage form. The developed microemulsion gel formulation was efficacious for the delivery of lipophilc and poorly soluble drugs such as lacidipine. The results demonstrated that the formulation was nonirritating and did not cause any erythyma upon transdermal administration (Kreilgaard et al., 2000; Vyas et al., 2004).

\section{Other nanomaterials}

(a) Nanotubes- They are hallow cylinders made of carbon atoms. They can also be filled and sealed, forming test tubes or potential drug delivery devices. (b) Nanowires- Glowing silica nano wire is wraped around a single stand of human hair. It looks delicate. It is about five times smaller than virus applications for nano wires include the early sensing of breast and ovarian malignancies.

(c) Nanocantilever- The honey comb mesh behind this tiny carbon cantilever is surface of fly's eye. Cantilevers are beams anchored at only one end. In nano world they function as sensors ideal for detecting the presence of extremely small molecules in biological fluid.

(d) Nanoshells- Nanoshells are hollow silica spheres covered with gold. Scientists can attach antibodies to their surfaces enabling the shells to target certain shells such as cancer cells. Nano shells one day also are filled with drug containing polymers.

(e) Quantum dots- Quantum dots are miniscule semiconductor particles that can serve as sign pots of certain type of cells or molecules in the body. They can do this because they emit different wavelengths of radiations depending upon the type of cadmium used in their cores. Cadmium sulphide for ultraviolet to blue, cadmium selinide for most of the visible spectrum and cadmium telluride for far infra red and near infra red.

(f) Nano pores- Nano pores have cancer research and treatment applications. Engineered into particles, they are holes that are so tiny that DNA molecules can pass through them one strand at a time allowing for highly precise and efficient DNA sequencing. By engineering nanopores into surface of drug capsule that are only slightly larger than medicines molecular structure, drug manufacturers can also use nanopores to control rate of drug's diffusion in body.

(g) Gold nanoparticles- These nanoparticles seen in transmission electron micrograph image, they have solid core. Researchs at north western university are using gold particles to develop ultra sensitive detection systems for DNA and protein markers associated with many forms of cancer including breast, prostate cancer.

(h) Bucky balls- Bucky ball is common for a molecule called buckminsterfullerene, which is made of 60 carbon atoms formed in shape of hollow ball discovered in 1985 . Bucky balls and other fullerenes because of their chemistry and their unusual hollow cage like shape extremely stable and can withstand high temperatures.

(i) Microspheres: Microspheres are characteristically free flowing powders consisting of proteins or synthetic polymers which are biodegradable in nature and ideally having a particle 
size less than $200 \mu \mathrm{m}$. Materials used for preparing Microspheres are polymers.

\section{Conclusion}

Delivery of therapeutics by nanocarriers demonstrates clear advantages over free non-bound drugs in terms of pharmacokinetics, body distribution, limitation of adverse side effects and therapeutic efficacy. Moreover, targeting of drug carriers specifically to diseased organs, tissues and cells further enhances their organ distribution and therapeutic potential while limiting adverse side effects upon healthy tissues. Furthermore, it was found that such targeting practically nivellates differences in the body distribution and therapeutic efficacy between these nanoparticles. In addition to characteristics of nanoparticles and their targeting, the type of route of administration plays an important role in the efficacy and adverse side effects of treatment. While systemic delivery of non-targeted systems in most cases is accompanied by substantial adverse side effects, local delivery limits such effects whereas improves the treatment. Likewise, local delivery of targeted therapeutics (e.g. local pulmonary delivery of nanoparticles for treatment of diseases) combines the advantages of both approaches and further enhances the efficacy of the treatment and limits its adverse side effects. Nevertheless, some issues in formulation, toxicity, targeting, safety, efficacy and storage stability of nanoparticles need to be addressed in further investigations.

\section{Conflict of interest: None}

\section{References}

Alibakhshi AF, AbarghooiKahaki S, Ahangarzadeh H, Yaghoobi F, Yarian R, Arezumand J, Ranjbari A, Mokhtarzadeh M. de la Guardia. 2017. Targeted cancer therapy through antibody fragments-decorated nanomedicines, Journal of Controlled Release, 268:323-334.

Arunachalam A, karthikeyan M, Vinay Kumar D. 2010.Transdermal drug delivery system:a review. Current Pharma Research, 1(1):70-81.

Bardal SK, Waechter JE, Martin DS. 2011. Pharmacokinetics, in: S.K. Bardal, J.E. Waechter, D.S. Martin (Eds.) Applied Pharmacology, Philadelphia, pp. 17-34.

Bulbake U, Doppalapudi S, Kommineni N, Khan W. 2017. Liposomal formulations in clinical use: an updated review. Pharmaceutics, 9:2-55.

Chen AM, Taratula O, Wei D, Yen HI, Thomas T, Thomas TJ, Minko T, He H. 2010. Labile catalytic packaging of DNA/siRNA: control of gold nanoparticles "out" of DNA/siRNA complexes. ACS Nano, 4:3679-3688.

Cruz E, Kayser V. 2019. Monoclonal antibody therapy of solid tumors: clinical limitations and novel strategies to enhance treatment efficacy. Biologics, 13:33-51.

Dhawan D, Ramos-Vara JA, Naughton JF, Cheng L, Low PS, Rothenbuhler R, Leamon CP, Parker N, Klein PJ, Vlahov IR, Reddy JA, Koch M, Murphy L, Fourez LM, Stewart JC, Knapp DW. 2013. Targeting folate receptors to treat invasive urinary bladder cancer. Cancer Research, 73:875-884.

Duong T, Li X, Yang B, Schumann C, Albarqi HA, Taratula O, Taratula O. 2017.Phototheranosticnanoplatform based on a single cyanine dye for image-guided combinatorial phototherapy. Nanomedicine, 13:955-963.

Engel JB, Tinneberg HR, Rick FG, Berkes E, Schally AV. 2016. Targeting of peptide cytotoxins to lhrh receptors for treatment of cancer. Current Drug Targets, 17:488-494.

Fliervoet LAL, Mastrobattista E. 2016. Drug delivery with living cells. Advanced Drug Delivery Review, 106:63-72.

Garbuzenko OB, Winkler J, Tomassone MS, Minko T. 2014.Biodegradable Janus nanoparticles for local pulmonary delivery of hydrophilic and hydrophobic molecules to the lungs. Langmuir, 30:12941-12949.

Garg A, Garg S, Sahu NK, Rani S, Gupta U, Yadav AK. 2019. Heparin appended ADH-anionic polysaccharide nanoparticles for sitespecific delivery of usnic acid. International Journal of Pharmaceutics, 557:258-253.

Garg A, Sharma R, Pandey V, Patel V, Yadav AK. 2017. Heparin-Tailored Biopolymeric Nanocarriers in SiteSpecific Delivery: A Systematic Review. Critical Reviews ${ }^{\mathrm{TM}}$ in Therapeutic Drug Carrier Systems, 34:1-33.

Gil PR, Hühn D, Mercato LLD, Sasse D, Parak WJ. 2010. Nanopharmacy: inorganic nanoscale devices as vectors and active compounds. Pharmacological Research, 62:115-25.

Han SJ, Tang J, Kumar B, Falk A, Farmer D, Tulevski G, Jenkins K, Afzali, A, Oida S. Ott J, Hannon J, Haensch W. 2017. High-speed logic integrated circuits with solutionprocessed self-assembled carbon nanotubes. Nature Nanotechnology, 12:861-865.

Kodama RH. 1999. Magnetic nanoparticles. Journal of Magnetism and Magnetic Materials 200:359-72.

Kreilgaard M, Pedersen EJ, Jaroszewski JW. 2000. NMR characterisation and transdermal drug delivery potential of microemulsion systems. Journal of Controllled Release, 69:421-433.

Vyas SP, Khar RK. 2004. Targeted and controlled drug delivery. 1:417-425.

Laurent S, Forge D, Port M, Roch A, Robic C, Vander Elst L. 2008. Magnetic iron oxide nanoparticles: synthesis, 
stabilization, vectorization, physicochemical characterizations, and biological applications. Chemical Reviews, 108:2064-110.

Ling D, Lee N, Hyeon T. 2015. Chemical synthesis and assembly of uniformly sized iron oxide nanoparticles for medical applications. Accounts of Chemical Research, 48:1276-85.

Maeda H. 2017. Polymer therapeutics and the EPR effect. Journal of Drug Target, 25:781.

Mainardes RM, Silva LP. 2004. Drug delivery systems: past, present, and future. Current Drug Targets, 5:449-455.

Mejia Oneto JM, Khan I, Seebald L, Royzen M. 2016. In VivoBioorthogonal Chemistry Enables Local Hydrogel and Systemic Pro-Drug To Treat Soft Tissue Sarcoma. ACS Central Science, 2:476-482.

Mendes LP, Sarisozen C, Luther E, Pan J, Torchilin VP. 2019. Surface-engineered polyethyleneimine-modified liposomes as novel carrier of siRNA and chemotherapeutics for combination treatment of drug-resistant cancers. Drug Delivery, 26:443-458.

Minko T, Dharap SS, Pakunlu RI, Wang Y. 2004.Molecular targeting of drug delivery systems to cancer. Current Drug Targets, 5:389-406.

Minko T. 2013. Nanotechnology and drug resistance. Advanced Drug Delivery Review, 65:1665-1666.

Orel V, Shevchenko A, Romanov A, Tselepi M, Mitrelias T, Barnes CH. 2015.Magnetic properties and antitumor effect of nanocomplexes of iron oxide and doxorubicin. Nanomedicine: Nanotechnology, Biology, and Medicine, $11: 47-55$.

Panyam J, Labhasetwar V. 2003. Biodegradable nanoparticles for drug and gene delivery to cells and tissue. Advanced Drug Delivery Review, 55:329-347.

Robinson DH, Mauger JW, 1991. Drug delivery systems. American Journal of Hospital Pharmacy, 48:S14-23.

Saad M, Garbuzenko OB, Ber E, Chandna P, Khandare JJ, Pozharov VP, Minko T. 2008. Receptor targeted polymers, dendrimers, liposomes: which nanocarrier is the most efficient for tumor-specific treatment and imaging?. Journal of Controlled Release, 130:107-114.

Sahle FF, Metz H, Wohlrab J, Neubert RH. 2013. Lecithin-based microemulsions for targeted delivery of ceramide AP into the stratum corneum: formulation, characterizations, and in vitro release and penetration studies. Pharmaceutical Research, 30:538-551.

Savla R, Taratula O, Garbuzenko O, Minko T. 2011 Tumor targeted quantum dotmucin 1 aptamer-doxorubicin conjugate for imaging and treatment of cancer. J Controlled
Release, 153:16-22.

Sharma A. 1997. Liposomes in drug delivery: Progress and limitations. International Journal of Pharmaceutics, 154:123-140.

Sharma V, Park K, Srinivasarao M. 2009. Colloidal dispersion of gold nanorods: historical background, optical properties, seed-mediated synthesis, shape separation and self-assembly. Materials Science and Engineering: R: Reports, 65:1-38.

Taratula O, Garbuzenko OB, Kirkpatrick P, Pandya I, Savla R, Pozharov VP, He H, Minko T. 2009. Surfaceengineered targeted PPI dendrimer for efficient intracellular and intratumoralsiRNA delivery. J Controlled Release, 140:284-293.

Taratula O, Kuzmov A, Shah M, Garbuzenko OB, Minko T. 2013. Nanostructured lipid carriers as multifunctional nanomedicine platform for pulmonary co-delivery of anticancer drugs and siRNA. Journal of Controlled Release, 171:349-357.

Taratula O, Savla R, Minko T. 2011. Poly(propyleneimine) dendrimers as potential siRNA delivery nanocarrier: from structure to function. International Journal of Nanotechnology, 8:36-52.

Wan X, Beaudoin JJ, Vinod N, Min Y, Makita N, Bludau H, Jordan R, Wang A, Sokolsky M, Kabanov AV. 2019. Codelivery of paclitaxel and cisplatin in poly(2-oxazoline) polymeric micelles: Implications for drug loading, release, pharmacokinetics and outcome of ovarian and breast cancer treatments. Biomaterials, 192:1-14.

Wayne R. Gombotz, in Biomaterials Science (Third Edition), 2013

Xie J, Chen K, Huang J, Lee S, Wang J, Gao J. 2010. PET/NIRF/MRI triple functional iron oxide nanoparticles. Biomaterials, 31:3016-22.

Zhang M, Garbuzenko OB, Reuhl KR, RodriguezRodriguez L, Minko T. 2012. Two in- one: combined targeted chemo and gene therapy for tumor suppression and prevention of metastases. Nanomedicine (Lond), $7: 185-197$.

Zheng T, Bott S, Huo Q. 2016. Techniques for accurate sizing of gold nanoparticles using dynamic light scattering with particular application to chemical and biological sensing based on aggregate formation. ACS Applied Materials and Interfaces, 8:21585-94. 